NRCPS-HE-99-13

NTUA-99/19

\title{
Vacuum Structure of Gauge Theory on Lattice with Two Parallel Plaquette Action ${ }^{\circ}$
}

\author{
K.Farakos \\ Physics Department, National Technical University, \\ Zografou Campus, 15780 Athens, Greece \\ email:kfarakos@central.ntua.gr \\ G.K.Savvidy \\ National Research Center Demokritos, \\ Ag. Paraskevi, GR-15310 Athens, Greece \\ email: savvidy@argo.nrcps. ariadne-t.gr
}

\begin{abstract}
We perform Monte Carlo simulations of a lattice gauge system with an action which contains two parallel plaquettes. The action is defined as a product of gauge group variables over two parallel plaquettes belonging to a given three-dimensional cube. The peculiar property of this system is that it has strong degeneracy of the vacuum state inherited from corresponding gonihedric $Z_{2}$ gauge spin system. These vacuua are well separated and can not be connected by a gauge transformation. We measure different observables in these vacuua and compare their properties.
\end{abstract}

\footnotetext{
${ }^{1}$ PACS 11.15.Ha 12.40.Ee 11.25.Pm
} 


\section{Introduction}

In this article we will report some observation that we have made in gauge invariant model defined on a lattice with an action which contains two parallel plaquettes [2]. This action is non-Abelian generalization of the $Z_{2}$ gauge spin system which was introduced as a lattice realization of random surfaces with gonihedric action [5]. It is defined as a product of gauge group variables over two parallel plaquettes belonging to a given three-dimensional cube of the lattice [2]

$$
H_{\text {gonihedric }}=\frac{n}{g^{2}} \sum_{\{\text {parallel plaquettes }\}}\left\{1-\left(1 / n^{2}\right) \operatorname{Re} \operatorname{Tr} U_{\text {plaq }} \cdot \operatorname{Re} \operatorname{Tr} U_{\text {plaq }}\right\},
$$

where $U_{\text {plaq }}$ is a product of gauge group elements $U_{i j}$ defined on a link $\langle i, j\rangle$ over all sites of a plaquette and $U_{i j}=\exp \left(i g A_{\mu} a\right)$. As one can see the action contains a product of two traces taken over two parallel plaquettes belonging to a $3 \mathrm{D}$-cube. The summation is over all pairs of parallel plaquettes 2 .

It is easy to see that for smooth classical fields the action reduces to the original action for non-Abelian fields as it takes place for the Wilson action [4, 1]

$$
H=\frac{2 n}{g^{2}} \sum_{\{\text {plaquettes }\}}\left(1-(1 / n) \operatorname{Re} \operatorname{Tr} U_{\text {plaq }}\right) .
$$

Indeed the matrix product of four $U^{\prime} s$ around plaquette $\mu, \nu=1,2$ becomes

$$
\operatorname{Re} \operatorname{Tr} U_{\text {plaq }}=\operatorname{Re} \operatorname{Tr} \exp \left(i g a^{2} F_{12}+\ldots\right)
$$

and after expanding fields around the center $x_{\mu}$ of the 3D-cube we will get

$$
1-\left(1 / n^{2}\right)\left(n-\frac{g^{2} a^{4}}{2} \operatorname{Tr} F_{12}^{2}\left(x_{\mu}+\frac{a}{2} \delta_{\mu 3}\right)\right)\left(n-\frac{g^{2} a^{4}}{2} \operatorname{Tr}_{12}^{2}\left(x_{\mu}+\frac{a}{2} \delta_{\mu 3}\right)\right) \approx \frac{g^{2} a^{4}}{n} \operatorname{Tr} F_{12}^{2}
$$

therefore it reproduces the classical action for pure Yang-Mills fields.

Thus on a classical level both models (1) and (2) are equivalent. But on a quantum level it is not obvious at all. The reason is that the vacuum structure of the model (11) is drastically different [2, 7]. In addition to the trivial vacuum configuration when all plaquette variables are equal to one

$$
\frac{1}{2} \operatorname{Tr} U_{\text {plaq }}=+1
$$

there are $2^{d N}$ different vacuum configurations [2, 7] with frustrated plaquettes $\frac{1}{2} \operatorname{Tr} U_{\text {plaq }}=$ -1 . One of these vacuua can be easily described as a configuration with all plaquettes frustrated

$$
\frac{1}{2} \operatorname{Tr} U_{\text {plaq }}=-1
$$

It is obvious that it is impossible to connect vacuum configurations (3) and (4) by a gauge transformation. The riach vacuum structure inherited from the "parent" $Z_{2}$ gauge spin system with the Hamiltonian [2]

$$
H_{\text {gonihedric }}=-\frac{1}{g^{2}} \sum_{\{\text {parallel plaquettes }\}}(\sigma \sigma \sigma \sigma)(\sigma \sigma \sigma \sigma)
$$

\footnotetext{
${ }^{2}$ The model with an action which is defined as a sum of contributions over all closed loops made up with six non-repeated links has been considered in [6].
} 
In both models (5) and (11) we have the same vacuum structure: towers of frustrated plaquettes describe different vacuua [7]. The difference between $S U(2)$ and $Z_{2}$ systems is that in the model (1) we have a continuous Lie gauge group and therefore continuous fluctuations of gauge fields around these vacuua.

The problem of our main concern is the physics in these vacuua and its relevance to the continuum limit. It is also interesting to understand: what is the remnant of this riach vacuum structure in the continuum limit. Is there any dilaton-like field which describes different vacuua in the continuum limit or not? And if there is a continuum limit in all these vacuua, then what is the difference between them? In the next section we will present results of simple analytical consideration of the model and the results of the Monte Carlo simulations for the $S U(2)$ gauge group which are performed on three-dimensional lattices for an obvious simplification [8, 9].

\section{High and low temperature expansion of average action}

As usually, all observables can be constructed as a product of gauge variables over closed loops and we shall consider first the average action. For the Wilson action (2) a simple one-plaquette average is equal to [1]

$$
P=<1-\frac{1}{2} \operatorname{Re} \operatorname{Tr} U_{\text {plaq }}>=1-\frac{\beta}{4}+\ldots \text { when } \quad \beta \rightarrow 0
$$

and

$$
P=\frac{3}{4 \beta}+\ldots \quad \text { when } \quad \beta \rightarrow \infty .
$$

Let us now consider two-plaquette action (1) which, as we shall see, has a different high temperature expansion. At high temperatures the average action is equal to:

$$
P P=<1-\frac{1}{4} \operatorname{Re} \operatorname{Tr} U_{\text {plaq }} \cdot \operatorname{Re} \operatorname{Tr} U_{\text {plaq }}>=1-\frac{\beta}{16}+\ldots
$$

and the low temperature expansion is the same as for the standard action

$$
P P=\frac{3}{4 \beta}+\ldots
$$

On Figure 1 one can see the behavior of average action in both models which we got by Monte Carlo simulations. In the simulations we used the Heat Bath algorithm [11]. The new action has indeed smaller values at high temperatures and the slope is four times smaller.

One can see also crossover from high to low temperatures. In the thermal cycles we have seen small hysteresis loop, but the measurement of susceptibility demonstrates that the maximum does not scale with the volume ( see Figure 2,3). Thus we do not see any phase transition at $\beta \approx 3.5$. 


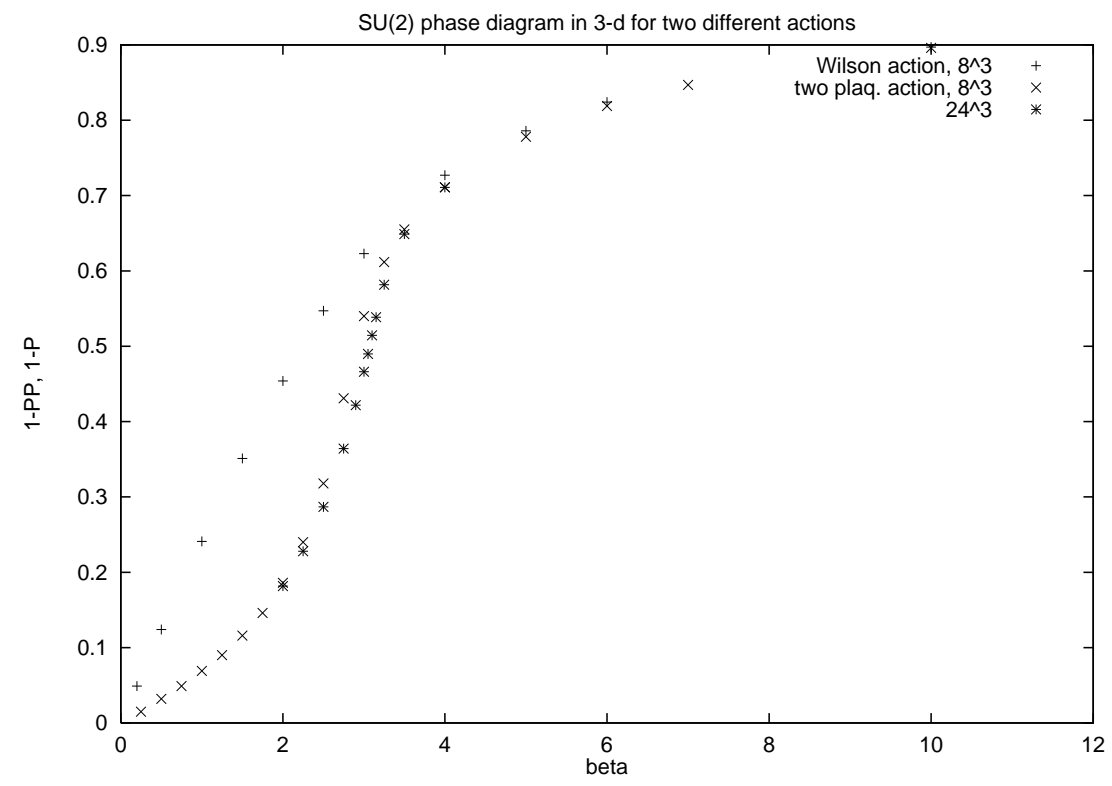

Figure 1: The behaviour of the average action in two different models. The new action has four times smaller values at high temperatures.

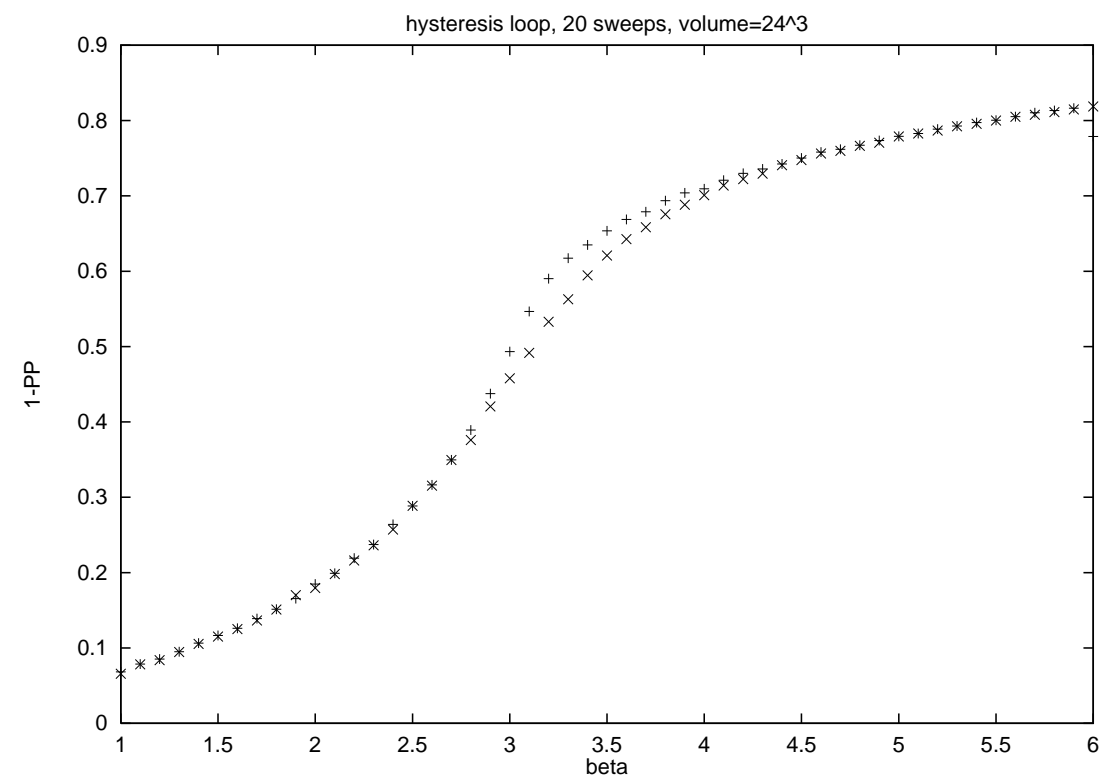

Figure 2: The thermal cycle of the two-plaquette action. 


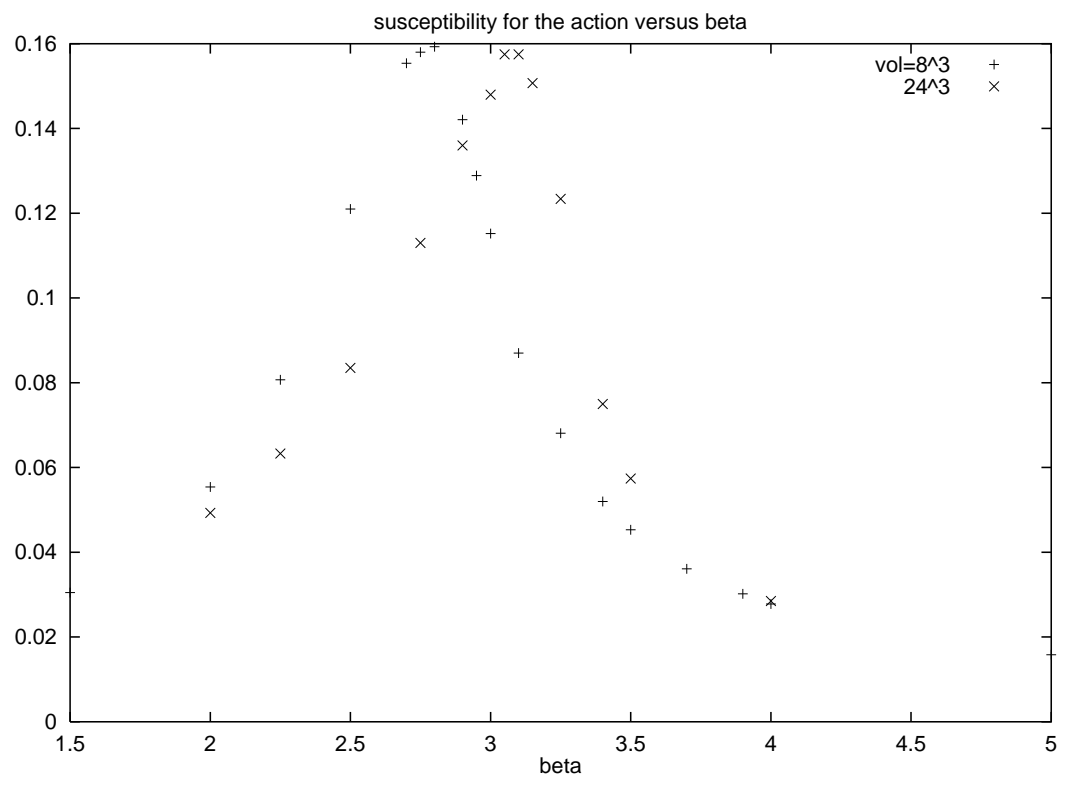

Figure 3: Susceptibility versus $\beta$ for two volumes $8^{3}, 24^{3}$. The maximum of susceptibility does not scale with the volume.

\section{Loop averages and vacuum structure}

In the model with a one-plaquette action (2) the high temperature expansion of the loop averages is equal to [1]

$$
<W(C)>=W(I, J)=\left(\frac{\beta}{4}\right)^{I J}, \quad \beta \rightarrow 0,
$$

and if one defines $W(I, J) \approx \exp (-\sigma A)$, where $A$ is the area $I J$ of the loop $C$ then the string tension is equal to

$$
\sigma \approx \frac{1}{a^{2}} \ln \left(\frac{4}{\beta}\right)
$$

In the two-plaquette model (1) the loop averages demonstrate peculiar behaviour. Indeed at high temperature the loop average

$$
<W(C)>=Z^{-} \cdot \int d U e^{-S} \frac{1}{2} \operatorname{Tr}_{C} U_{i j}
$$

is equal to zero for $S U(2)$ gauge group, because there are no low order lattice diagrams (proportional, let us say, to area) which can contribute to the expectation value of $W(C)$ (see Figures 4 and 5). We have absolute confinement in this model. It is similar to the limit $n \rightarrow \infty$ of the model with one-plaquette action

$$
\sigma=\frac{1}{a^{2}} \ln \frac{2 n^{2}}{\beta} \rightarrow \infty
$$

and thus $\langle W(C)>=\approx \exp (-\sigma A) \rightarrow 0$.

\footnotetext{
${ }^{3}$ The situation changes for large gauge groups where there exist high temperature diagrams which contribute to one-plaquette average. Specifically for the $S U(3)$ group the 3D-box diagram on a top of plaquette gives nonzero contribution.
} 


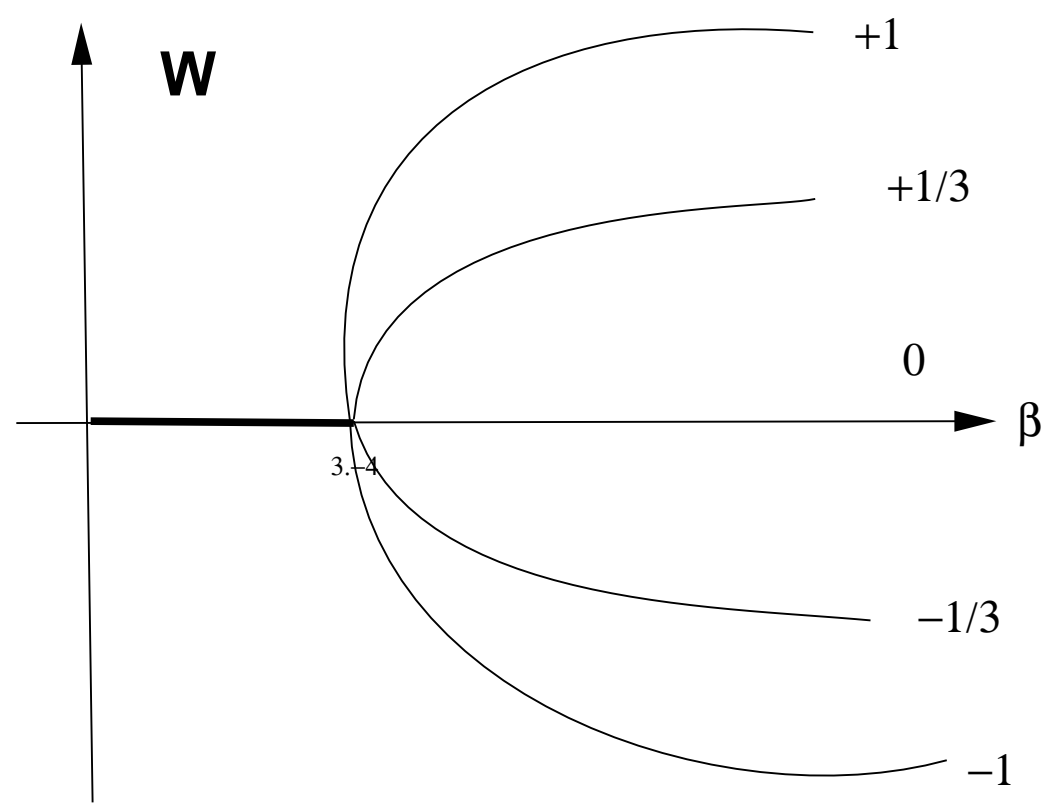

Figure 4: Schematic multivacuum structure in the model with two-plaquette action. This figure shows the absolute confinement at high temperatures $\beta \leq 3.5$ and gauge nonequivalent vacuua at low temperatures.

The Monte-Carlo simulations of $S U(2)$ group demonstrate that this behaviour takes place until $\beta \approx 3.5-4$ as one can see on Figures 4 and 5 . At these temperatures there appears proliferation of large lattice diagrams which cover almost the whole lattice and $<W(C)>$ gets nonzero value.

At low temperatures the nontrivial vacuum structure begins to play a central role. To see that let us consider the situation when two opposite plaquettes inside a 3D cube are frustrated. As it is easy to see from the action the energy of the new configuration will be the same. Indeed the energy is minimal when $\frac{1}{4} \operatorname{Tr} U_{\text {plaq }} \cdot \operatorname{Tr} U_{p l a q}^{\text {paral }}=1$, therefore we have two possibilities ether

$$
\frac{1}{2} \operatorname{Tr} U_{\text {plaq }}=\frac{1}{2} \operatorname{Tr} U_{\text {plaq }}^{\text {paral }}=+1
$$

or

$$
\frac{1}{2} \operatorname{Tr} U_{\text {plaq }}=\frac{1}{2} \operatorname{Tr} U_{\text {plaq }}^{\text {paral }}=-1
$$

On the whole lattice one can build towers of frustrated plaquettes so that the new configuration will have the same energy as the trivial vacuum $\frac{1}{2} \operatorname{Tr} U_{\text {plaq }}=+1$. Thus the vacuum has global degeneracy as it was in the corresponding $Z_{2}$ gauge spin system [2]. This actually means that we have many vacuua in which the gauge field $A_{\mu}$ is very far from perturbative value.

This complicated vacuum structure is reflected on Figures 4 and 5 which show multivacuum structure at low temperature. The top curve corresponds to the vacuum in which all plaquettes are equal to plus one, $\frac{1}{2} \operatorname{Tr} U_{\text {plaq }}=1$, and the bottom curve corresponds to the opposite case when all plaquettes are frustrated $\frac{1}{2} \operatorname{Tr} U_{\text {plaq }}=-1$. It is also not difficult to construct explicitly vacuum configurations in which only part of the lattice plaquettes are frustrated. The vacuum with all plaquettes frustrated, $\frac{1}{2} \operatorname{Tr} U_{\text {plaq }}=-1$, corresponds to a gauge field which is far from the perturbative value $A_{\mu}=0$ in the whole lattice. 


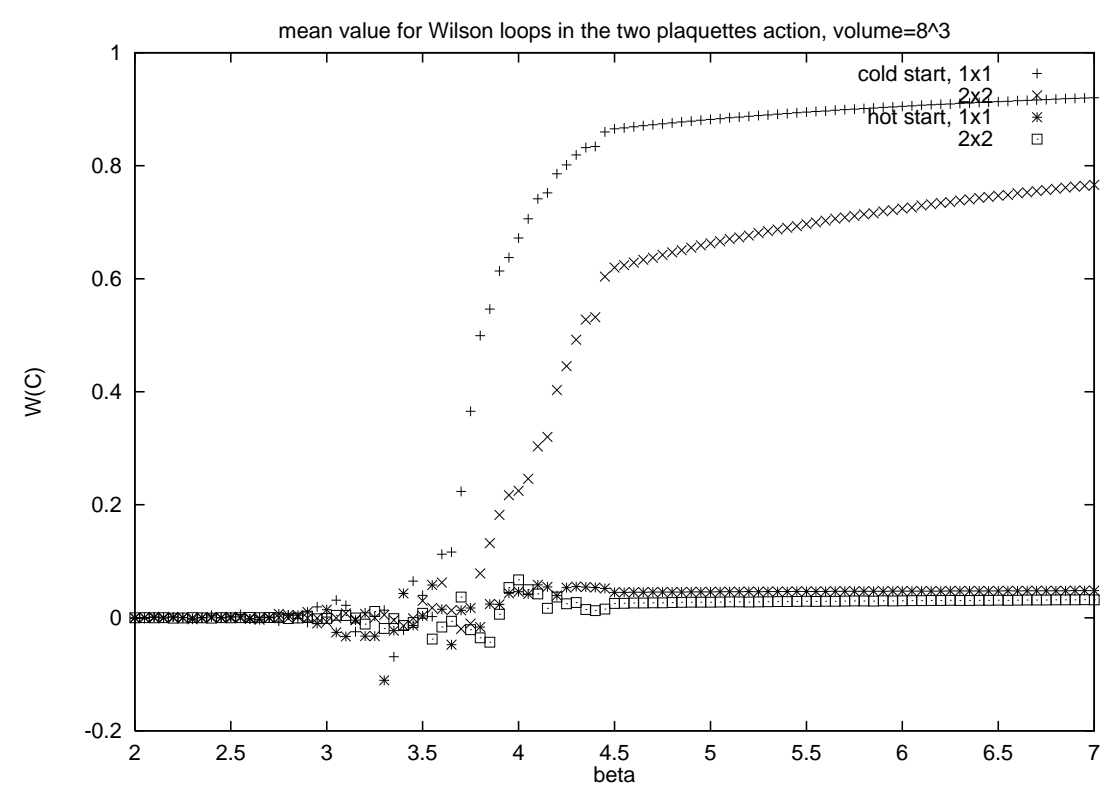

Figure 5: The behaviour of the mean value for the $1 \times 1$ and $2 \times 2$ loops for two-plaquette action. It represents the evolution of the averages starting from low temperature with $\frac{1}{2} \operatorname{Tr} U_{\text {plaq }}=+1$ to high temperature and then back to low one. As one can see the system fluctuates at pseudocritical point and then falls into vacuum with $\operatorname{Tr} U_{\text {plaq }} \approx 0$

Two natural questions arize:

i) is there a sensible continuum limit in all these vacuua in the limit $a \rightarrow 0, \beta \rightarrow \infty$ ?

ii) and if there is a continuum limit, then what is difference between them ? What type of parameter ("coupling constant") or field ("dilaton") may describe them?

\section{String tension in different vacuua}

In this paragraph we will study the behaviour of string tension in the limit $\beta \rightarrow \infty$. We choose this quantity because it can characterize the system in the continuum limit if it exists. As usually we define the string tension by the behaviour of $\mathrm{W}(\mathrm{L}, \mathrm{L})$ for large $\mathrm{L}$ as:

$$
W(L, L) \approx e^{-\left(\sigma L^{2}+m L+C o n s t\right)}
$$

To calculate string tension we shall follow the procedure:

i) calculate $\mathrm{W}(\mathrm{L}, \mathrm{L})$ for different values of $L \leq 6$ at a given $\beta$ (in our simulations the lattice volume is $16^{3}$ and the coupling constant is in the range $6<\beta<10$ ), ii)then fit the quantity $-\ln |W(L, L)|$ using second order polynomials and extract $\sigma$ for each $\beta$,

iii)because string tension scales as $\sigma=\sigma_{\text {phys }} a^{2}$ and $\beta=2 / g^{2} a$ we shall plot the ratio $\beta \sqrt{\sigma}=2 \sqrt{\sigma_{\text {phys }}} / g^{2}$ versus $1 / \beta$ (see Figure 6 ).

The last ratio converges to a finite limit when $\beta \rightarrow \infty$. We expect that this limit can be reached within the finite size corrections which are of order $O\left(\frac{1}{\beta}\right)$. On Figure 6 we also depicted string tension in the vacuum $<\frac{1}{2} \operatorname{Tr} U_{\text {plaq }}>=+1$ calculated for the larger lattice $24^{3}$ in order to see the variation with volume. The convergence is better for small $\beta$. 


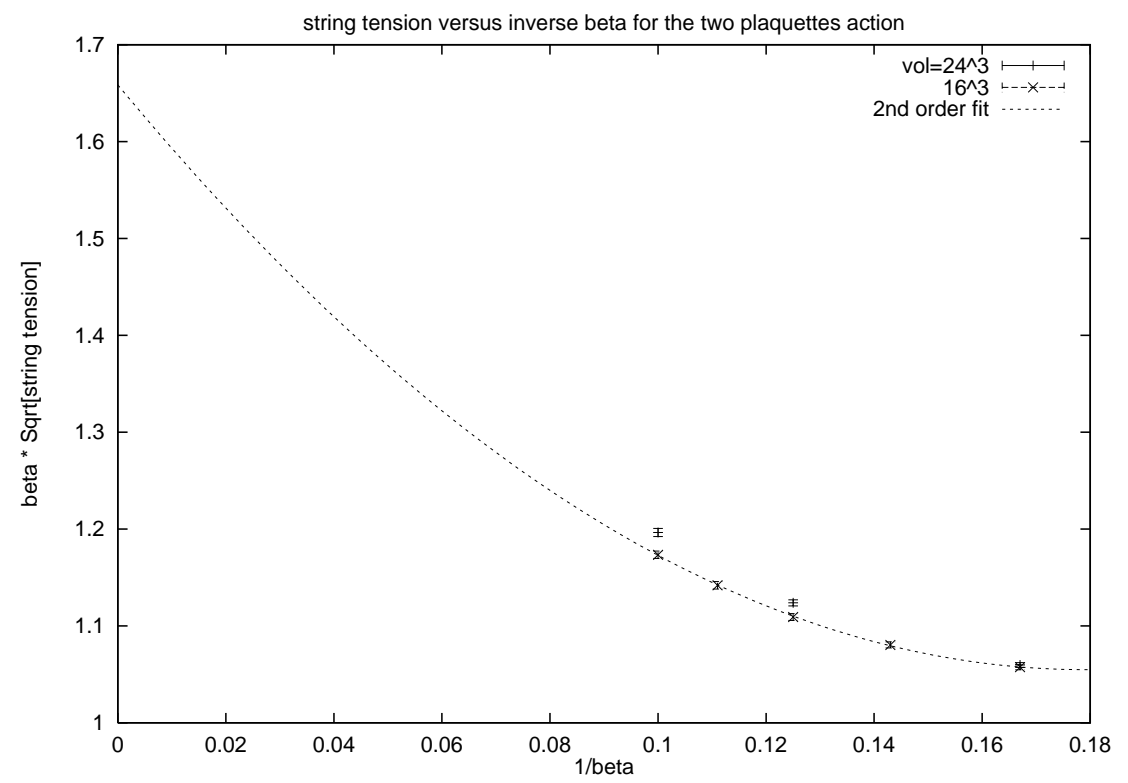

Figure 6: String tension versus inverse $\beta$ in the standard vacuum $\operatorname{Tr} U_{\text {plaq }}=+1$.

Until now we have been calculating string tension in the vacuum $<\frac{1}{2} \operatorname{Tr} U_{\text {plaq }}>=$ +1 when $\beta \rightarrow \infty$. In the new vacuua we have frustrated plaquettes and the quantity $\frac{1}{2} \operatorname{Tr} U_{\text {plaq }}$ is less than one. It remains constant during Monte Carlo simulations for large $\beta \geq 6$ and we conclude that these vacuua are well separated ft.

To explore vacuum structure we have to calculate $W(L, L)$ for these vacuua as well. A typical result is shown in Table 1 , where we choose $\beta=10$, the volume is equal to $16^{3}$ and $L$ is smaller than six. By "vacuum $-1 / 3$ " we mean the vacuum which has the mean value $<\frac{1}{2} \operatorname{Tr} U_{\text {plaq }}>=-1 / 3$.

We observe that the absolute value $|W(L, L)|$ is the same for two vacuua +1 and -1 , as well for the vacuua $+1 / 3$ and $-1 / 3$. The absolute value $|W(L, L)|$ for odd $L$ in the vacuua $+1 / 3$ or $-1 / 3$ is one third of its value in the vacuua +1 or -1 . Because of this relation, $\left|W_{1}(L, L)\right|=\frac{1}{3}\left|W_{1 / 3}(L, L)\right|$ we have ?

$$
-\ln \left|W_{1 / 3}(L, L)\right|=-\ln \left|W_{1}(L, L)\right|+\ln (3)=\sigma L^{2}+m L+\ln (3)+\text { Const }
$$

and the string tension is the same for all these vacuua. Thus we can argue that string tension does indeed scale in all these vacuua and we can speculate that one can distinguish them by the scalar operator $\frac{1}{2}<\operatorname{Tr} U_{\text {plaq }}>$-gluon like condensate [12].

One of us (K.F.) wishes to thank the EU for partial financial support (TMR project FMRX-CT97-0122).

\section{TABLE 1}

The values of $\left\langle W(L, L)>\right.$ in different vacuua for $\beta=10$ and volume $16^{3}$ :

\footnotetext{
${ }^{4}$ When we start from a given vacuum configuration $<\frac{1}{2} \operatorname{Tr} U_{\text {plaq }}>\neq+1$ it never drifts into other vacuua when $\beta \geq 6$.

${ }^{5} \mathrm{By} W_{-1}(L, L)$ we mean the mean value of $\mathrm{W}(\mathrm{L}, \mathrm{L})$ in the vacuum $\operatorname{Tr} U_{\text {plaq }}=-1$ for example.
} 


\begin{tabular}{||c|c|c|c|c|}
\hline \hline $\mathrm{L}$ & vacuum +1 & vacuum -1 & vacuum $+1 / 3$ & vacuum- $1 / 3$ \\
\hline \hline 1 & $0.946281(4)$ & $-0.946277(3)$ & $0.315429(3)$ & $-0.315427(4)$ \\
\hline 2 & $0.83866(2)$ & $0.83863(2)$ & $0.83865(1)$ & $0.83864(2)$ \\
\hline 3 & $0.71894(4)$ & $-0.71889(4)$ & $0.23968(3)$ & $-0.23967(4)$ \\
\hline 4 & $0.60273(7)$ & $0.60265(8)$ & $0.60269(8)$ & $0.60265(9)$ \\
\hline 5 & $0.49675(13)$ & $-0.49656(12)$ & $0.16561(9)$ & $-0.16567(10)$ \\
\hline 6 & $0.40381(19)$ & $0.40352(20)$ & $0.40361(19)$ & $0.40369(21)$ \\
\hline \hline
\end{tabular}

\section{References}

[1] M.Creutz, Quarks, gluons and lattices. (Cambridge University Press,Cambridge 1983)

[2] G. K. Savvidy, K. G. Savvidy and F. J. Wegner. Nucl.Phys. B443 (1995) 565

[3] F.J. Wegner, J. Math. Phys.12 (1971) 2259

R.Balian, J.M.Drouffe and C.Itzykson. Phys.Rev. D10 (1974) 3376;

Phys.Rev. D11 (1975) 2098, 2104

[4] K.G.Wilson, Phys.Rev. D10 (1974) 2445

[5] G.K. Savvidy and K.G. Savvidy. Int. J. Mod. Phys. A8 (1993) 3993;

Mod.Phys.Lett. A8 (1993) 2963. R.V. Ambartzumian and et.al. Phys. Lett. B275 (1992) 99

[6] V.Azcoiti, G.Di.Carlo and E.Follana. Investigation of a toy model for frustration in Abelian lattice gauge theory. hep-lat/9803022

[7] G.K.Savvidy and F.J.Wegner. Nucl.Phys.B413(1994)605

G.K. Savvidy and K.G. Savvidy. Phys.Lett. B324 (1994) 72;

Phys.Lett. B337 (1994) 333; Mod.Phys.Lett. A11 (1996) 1379

R. Pietig and F.J. Wegner. Nucl.Phys. B466 (1996) 513;

Nucl.Phys. B525 (1998) 549

[8] K.Farakos,G.Koutsoumbas and S.Sarantakos. Phys.Lett. B189 (1987) 173

[9] M.Teper. Phys.Lett. B289 (1992) 115

[10] G. Koutsoumbas, G. Savvidy and K. Savvidy. Phys.Lett. B410 (1997) 241

[11] A.D.Kennedy, B.J.Pendleton Phys. Lett. B156 (1985) 393

[12] G.K.Savvidy. Phys.Lett. B78 (1977) 144

[13] M.Creutz, L. Jacobs and C. Rebbi. Phys.Rev.Lett. 42 (1979) 1390

G. Bhanot and M.Creutz Phys.Rev. D21 (1980) 2892 